\title{
Avaliação da Influência do Capital Intelectual nas Fases de Evolução de Empresas Startups de Base Tecnológica
}

\author{
Vanessa V. P. Canhete, Jefferson O. Gomes, Marcelo F. Prim, \\ Juliana G. Ulliana \& Germana A. B. Zapata
}

O crescimento do número de empresas startups de base tecnológica e sua contribuição para a introdução de novas tecnologias são reconhecidos pelo mercado e pela comunidade acadêmica como elementos de fortalecimento da inovação no país. $\mathrm{O}$ artigo apresenta a influência dos ativos intangíveis no sucesso destas empresas nascentes, sob a visão dos empresários deste mercado, nos primeiros anos de vida das startups. Para isso, realizou-se um levantamento survey com 15 empresários de empresas startups de base tecnológica, com o objetivo principal de avaliar a importância e/ou predominância de determinados tipos de capital intelectual, nas dimensões do capital humano, estrutural e relacional nos 5 primeiros anos de vida das empresas.

Palavras chaves: Startups de base tecnológica; Capital Intelectual.

The growing number of startups technology-based companies and their contribution to the introduction of new technologies are recognized by the market and by the academic community as elements of innovation strengthening in the country. This paper presents the entrepreneurs perspective about the the influence of intangible assets on the success of these new companies, in the early years of the startups. For this, it was conducted a survey methodology with entrepreneurs from 15 startups technology-based companies, with the main objective to assess the importance and / or prevalence of certain types of intellectual capital, in the dimensions of human, structural and relational capital, within the first 5 years of life companies.

Keywords: Startups technology-based; Intellectual Capital. 


\section{Introdução}

O crescimento do emprego, no século 21 , terá de vir de novos empreendimentos, de modo que toda a sociedade tenha interesse em promover um ambiente que a apoie no sucesso, crescimento e contratações. A criação de uma economia de inovação, que é impulsionada pela rápida expansão de empresas startups, nunca foi tão imperativa e desta forma, uma menor taxa de falha de empresas startups poderia trazer grandes consequências econômicas (BLANK, 2013).

Novas e pequenas empresas impactam positivamente na economia, no entanto, a falta de experiência empresarial, forte competição da indústria e a fragilidade são alguns dos mais citados argumentos que justificam o insucesso do negócio (PEÑA, 2002). Dada a importância que novos negócios representam para o desenvolvimento econômico e considerando que as empresas startups caracterizam uma abordagem atual para o desenvolvimento do empreendedorismo, por seu potencial de crescimento e abordagem tecnológica, tais empresas apresentam-se como importante fonte de pesquisa, incluindo os estudos sobre a influência das dimensões do capital intelectual sobre o sucesso empresarial (MACHADO, 2014).

Nesse sentido, em um mercado competitivo, a capacidade de uma empresa em criar e manter parceiras pode significar um fator crítico de sucesso. As organizações poderiam fazer maior uso de produtos e serviços fornecidos por outras organizações, caracterizando um ambiente propício para a colaboração e aprendizagem de novas práticas (WILLIAMSON, 1975).

\section{Revisão Bibliográfica}

\section{EMPRESAS STARTUPS}

Empresas nascentes de base tecnológica, as chamadas empresas startups, são empresas jovens e inovadoras, atuantes em quaisquer áreas de atividades cuja estratégia empresarial e de negócios é sustentada pela inovação e cuja base técnica de produção está sujeita a mudanças frequentes, advindas da concorrência centrada em esforços continuados de pesquisa e desenvolvimento tecnológico (REVISTA EXAME PME, 2015; FINEP, 2000).

Empresas startups utilizam-se de diversos tipos de inovação, sejam novas descobertas científicas, novas formas de uso de uma tecnologia existente, criação de um novo modelo de negócio, nova proposta de valor, novo segmento de clientes ou mesmo diferentes formas de disponibilização do produto (RIES, 2012).

Empresas startups poderiam melhorar seu desempenho inicial pelo estabelecimento de alianças, pela atuação em uma rede eficiente que possa prover acesso à informações diversas e recursos com custos mínimos de redundância, conflito e complexidade, e uma aliança com competidores estabelecidos que ofereçam mais oportunidades para o aprendizado e menor risco de rivalidade. Em empresas startups de alta tecnologia, como na área de biotecnologia, essas alianças podem ser particularmente eficazes para alavancar a inovação, embora em todos os tipos possam facilitar o acesso a recursos complementares, necessários para um crescimento bem sucedido (BAUM et al., 2010).

A Startup Enxuta (Lean Startup) é um método de lançamento de novas empresas baseado em falhas rápidas e valorização do aprendizado contínuo, favorecendo a experimentação em detrimento ao planejamento elaborado, atenção ao feedback do cliente em detrimento à intuição e o design interativo e colaborativo de produtos em detrimento aos modelos tradicionais de desenvolvimento de produto (RIES, 2012; BLANK, 2013; RIBEIRO, 2014).

Os principais fatores que restringem $\mathrm{O}$ desenvolvimento de empresas startups, são o alto custo de obtenção do primeiro cliente e do produto errado, os longos ciclos de desenvolvimento de tecnologia, o limitado número de pessoas dispostas a assumir os riscos inerentes à fundação e trabalho em empresas startup, a estrutura da indústria de capital de risco, em que poucas empresas faziam altos investimentos em poucas startups para aumentar as chances de retornos significativos e a concentração das competências reais na criação de startups em apenas alguns centros, em especial nos Estados Unidos (BLANK, 2013).

\section{FASES DE DESENVOLVIMENTO E EVOLUÇÃO DE UMA EMPRESA STARTUP}

Diversos autores apresentamas fases de desenvolvimento de uma empresa startup baseadas no fluxo de caixa ou no aporte de investimentos ao longo do tempo. Freeman e 


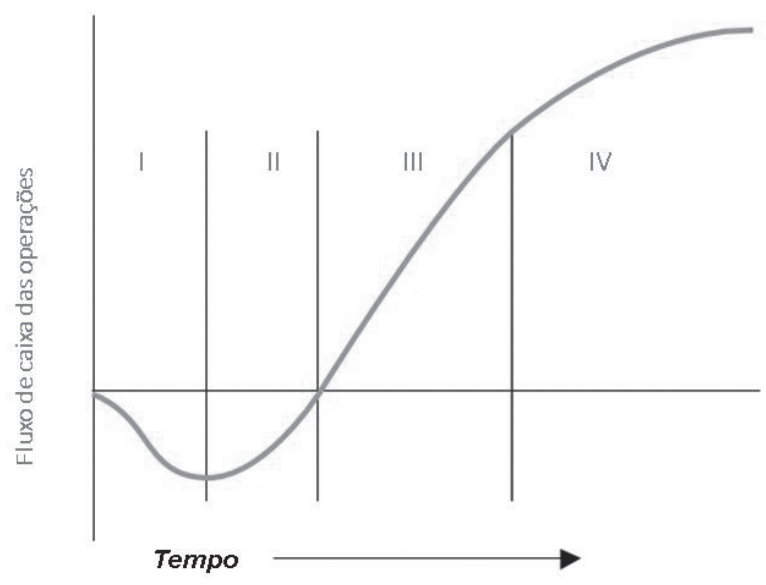

Figura 2.1. Evolução da empresa empreendedora (Fonte: Freeman \& Engel, 2007)

Engel (2007) sugerem quatro períodos de desenvolvimento de uma empresa iniciante, conforme figura 2.1.

Os estágios de evolução da empresa são marcados por eventos financeiros que motivam mudanças na estrutura organizacional e na gestão das atividades da empresa, e são caracterizados como (FREEMAN \& ENGEL, 2007; DORNELAS, 2008):

Fase da ideia ou Período de "Empreendedorismo puro": definição do conceito do negócio, obtenção dos recursos financeiros que são oriundos do próprio empreendedor, família, amigos ou de capitais anjos, formação da equipe, identificação de clientes, análise da concorrência, construção do protótipo, obtenção do primeiro contrato (cliente);

Fase de início ou Período de "Foco estratégico": conhecimento e implementação do negócio, clareza sobre o modelo de negócio, identificação de parceiros estratégicos, desenvolvimento de relacionamento com fornecedores, melhoramento do produto ou transição de um protótipo para um produto escalável, formação de uma equipe completa, levantamento de recursos próprios, a empresa já está constituída e, em geral, tem cerca de um ano de vida e o negócio ainda é considerado de alto risco;

Fase de expansão ou Período de "Construção dos sistemas": estabelecimento de controles financeiros, divisões no trabalho, relações de subordinação e autoridades, desenvolvimento de sistemas de controle interno, existência de sistemas operacionais (produção, terceirização, distribuição, vendas, serviços e garantias); geralmente tem de dois a três anos de existência e há necessidade de aumento de capital para acelerar o crescimento e com isso o início da entrada de investimentos de capital de risco;

Fase da consolidação ou Período da "Gestão empresarial": abertura de capital, formalização da cultura, racionalização da estratégia; fase de expansão e consolidação do negócio e possibilidade de abertura de capital.

Considerando as abordagens apresentadas, percebese que os três primeiros estágios são os que de fato correspondem ao período de gestação e abertura de novas empresas e período cuja estruturação adequada é fundamental para o amadurecimento e consolidação das mesmas no mercado.

\section{CAPITAL INTELECTUAL}

O termo Capital Intelectual é definido como o conhecimento existente de uma organização que é crítico para seu sucesso e pode influenciar a vantagem competitiva de uma organização, sendo um bem intangível (MERTINS et al, 2006; STEWART, 1998) .

Mertins, Alwert \& Will (2006) trazem a abordagem europeia da gestão do Capital Intelectual para pequenas e médias empresas, com adaptações à realidade brasileira, sob a forma de apresentação de lições aprendidas na implementação real do método ICS (Intellectual Capital Statement) em pequenas e médias empresas brasileiras do setor de óleo e gás. Pelo uso do método do ICS, é possível às pequenas e médias empresas aprender como melhor utilizar seus capitais humano, estrutural e relacional para melhorar suas próprias capacidades e assim se posicionarem de forma mais competitiva.

O projeto InCaS, Declaração do Capital Intelectual para a Europa (do inglês Intellectual Capital Statement for Europe) utiliza uma abordagem baseada na metodologia ICS alemã cuja diretriz descreve a estrutura e o conteúdo básico do ICS, bem como o modelo geral e o procedimento de criação do ICS. Dentro do processo de criação, a análise organizacional avalia e documenta o capital intelectual sob três dimensões: humano, relacional e estrutural.

Dentre os métodos de avaliação e gestão do capital 
intelectual (CI) destaca-se o projeto alemão "Wissensbilanz - Made in Germany", metodologia desenvolvida por meio de um consórcio liderado pelo Instituto Fraunhofer IPK em Berlin, na Alemanha, cuja aplicada foi voltada a pequenas e médias empresas alemãs (Federal Ministry of Economics and Labour, 2004) e baseada no InCaS. Mertins e Will (2009) sugerem que os resultados obtidos por meio da aplicação do método apresentam conclusões qualitativas e quantitativas sobre a relevância estratégica dos elementos do capital intelectual e suas variações, conforme o setor empresarial. Ainda segundo Mertins e Will (2009), o processo de avaliação do capital intelectual inicia-se com a visão e estratégia da organização, considerando o ambiente de negócios em que está inserida.

O Capital Intelectual pode ser dividido em três tipos (MERTINS, WILL, 2006):

- CAPITAL HUMANO: o capital humano refere-se ao conjunto de elementos dos recursos humanos ligados às habilidades, competências e motivação dos funcionários, sendo desta forma, uma propriedade dos mesmos, a incorporação da cultura e filosofia da empresa voltada ao atendimento;

- CAPITAL ESTRUTURAL: o capital estrutural abrange as estruturas e os processos da organização, que consiste nas estruturas inteligentes que são propriedade da organização, bem como sua tecnologia, invenções, dados, publicações geridos por ferramentas de gestão e processos definidos;

- CAPITAL RELACIONAL: o capital relacional consiste nas relações da organização com clientes, fornecedores, parceiros e a sociedade, na forma como apresenta e comercializa seus produtos e serviços e estabelece suas relações financeiras e contratuais.

Para cada um dos tipos de capital intelectual, são definidos fatores críticos que, então são avaliados, conforme as perspectivas de quantidade, qualidade e sistemática. Para Mertins e Will (2007), é fundamental a identificação dos fatores do capital intelectual, relevantes para os processos estratégicos de negócios e que agregam valor à empresa.

\section{RELAÇÃO DAS EMPRESAS STARTUPS E O CAPITAL INTELECTUAL}

A falta de experiência em negócios e a forte concorrência no setor exemplificam algumas das dificuldades enfrentadas por novas empresas para sobreviver em sua fase inicial, e os fatores de sucesso dessas empresas incluem tanto os fatores tangíveis como os intangíveis (PEÑA, 2002).

Peña (2002) definiu o processo de desenvolvimento de uma empresa startup em período "ex-ante", período de gestação e período de consolidação, identificando os elementos tangíveis e intangíveis de cada período. Sugere ainda que o sucesso de uma empresa startup pode ser, mesmo que de forma parcial, explicado pelo capital humano, pelo capital estrutural e pelos elementos de capital relacional, em que cada um desses fatores compõe os elementos intangíveis. Entre eles, as habilidades de empreendedores, o modo de tomada de decisões estratégicas, as adaptações ao mercado e a capacidade de trabalho em rede com parceiros externas.

Machado (2014) afirma que os ativos de capital intelectual desempenham um papel fundamental no crescimento e sobrevivência de novas empresas, realizando uma análise do grau de maturidade do capital intelectual em empresas startups incubadas e apresenta um modelo de influência do capital intelectual no sucesso de uma empresa startup. Um estudo realizado por Hormiga (2010), com 130 empresas startups, a fim de obter um melhor entendimento sobre a importância de ativos intangíveis em empresas recém-criadas, revelou que o capital humano tem forte influencia no desempenho das empresas no primeiro estágio de vida.

\section{Método da Pesquisa}

A proposta metodológica desenvolvida no trabalho baseou-se em uma pesquisa exploratória de abordagem qualitativa cujo método utilizado foi a pesquisa levantamento (survey) buscando conhecer as expectativas das empresas startups quanto ao capital intelectual. A avaliação da importância do capital intelectual, nas fases de evolução das empresas startups, seguiu as etapas:

1. Seleção das empresas startups e respectivos representantes;

2. Delimitação das fases de desenvolvimento das empresas startup;

3. Seleção dos tipos de capital intelectual e respectivos fatores de sucesso;

4. Construção do roteiro e aplicação do questionário; 
5. Análise dos dados e identificação do nível de importância do capital intelectual em cada fase de desenvolvimento.

Para esta pesquisa, o critério de seleção das empresas startups participantes da pesquisa foi a participação no Edital SENAI SESI de Inovação 2014, atendendo aos requisitos de faturamento anual de até $\mathrm{R} \$ 3,6$ milhões de reais e até cinco anos de existência. As fases de desenvolvimento de uma empresa startup consideradas neste trabalho foram baseadas nas definições da literatura a fim de garantir o mesmo entendimento a todos os entrevistados, e a delimitação seguiu o tempo de vida das empresas, estabelecendo três fases (períodos ou estágios) de evolução:

- FASE I: $1^{\circ}$ ano de vida da empresa (fase da ideia ou início)

- FASE II: do $2^{\circ}$ ao $3^{\circ}$ ano de vida da empresa (fase da prototipação, lançamento do produto)

- FASE III: do $3^{\circ}$ ao $5^{\circ}$ ano de vida da empresa (fase da consolidação)

Para a avaliação do capital intelectual foram consideradas as dimensões do capital humano, relacional e estrutural, com fatores de sucesso definidos na literatura por Mertins, Wang \& Will (2009) e aplicados em um estudo com pequenas e médias empresas, adicionaram-se ainda dois fatores de capital humano baseados nos conceitos Lean Startup apresentados por Ries (2012) quanto ao sucesso de uma empresa startup. Foi realizado o contato com os representantes das empresas startups, explicado o objetivo da pesquisa e o método de avaliação. A análise foi realizada para cada um dos fatores de sucesso nas fases de desenvolvimento da empresa, de acordo com as avaliações dos empresários cujas notas podiam variar de zero a cinco, no qual a nota zero significa que o fator não tem importância na fase da vida da empresa e para a nota cinco, o fator possui extrema importância na fase da vida da startup.

A análise dos dados consistiu na identificação dos fatores e tipos de capital intelectual prevalecentes em cada fase de evolução da empresa, segundo a percepção do grau de importância e necessidade de existência do capital intelectual pelas empresas, sendo que os empreendedores foram orientados quanto à necessidade de avaliação nas três fases, considerando sua visão acerca de seu momento atual, experiência em fases anteriores e perspectiva de futuro quanto à importância do capital intelectual.

A demonstração e análise dos resultados visou identificar os hiatos existentes entre as fases de evolução, permitindo assim uma avaliação da predominância do capital intelectual e as necessidades de ativos intangíveis e fatores mais importantes para as empresas startups ao longo de seus primeiros anos de vida, sob a ótica dos empresários.

\section{Análise e Interpretação dos Resultados}

A taxa de resposta com o retorno dos questionários preenchidos foi de $55,2 \%$, totalizando a participação de 16 empresas. Para avaliação dos resultados, foi calculada a média, a variância e o desvio padrão populacional dos dados.

A figura 4.1 apresenta os resultados das médias de cada fator do capital intelectual nas diferentes fases. Nota-se que na fase I, há a predominância dos fatores do capital humano na avaliação dos empresários, como fatores de maior importância para a empresa no primeiro ano de vida, com destaque para o fator $\mathrm{CH}-6$ "Capacidade de adaptação a mudanças", definido como a capacidade de aprender com os erros e de ter uma reação rápida a novos rumos da estratégia do negócio, redefinindo alternativas de mudanças ágeis sem alterar a essência da empresa.

Ainda na fase I, quanto ao capital estrutural e o capital relacional, destacam-se os fatores críticos de sucesso CE-4 "Inovação de Produtos" e CR-5 "Relacionamento com parceiros de cooperação", respectivamente. O fator "Inovação de Produtos" foi definido como as inovações significantes para o futuro da organização, caracterizadas por trazer o aprimoramento ou mudanças radicais aos produtos e/ou processos existentes e eventualmente, resultem em um pedido de patente. Já o fator "Relacionamento com parceiros de cooperação" refere-se às relações com as associações profissionais, organismos e sociedades, bem como a gestão desses relacionamentos, que compreende a aquisição conjunta de clientes, fornecedores, investidores, transferência de conhecimento em parcerias de Pesquisa \& Desenvolvimento, transferência de boas práticas e atividades em rede. 


\section{Artigo Geral 4}
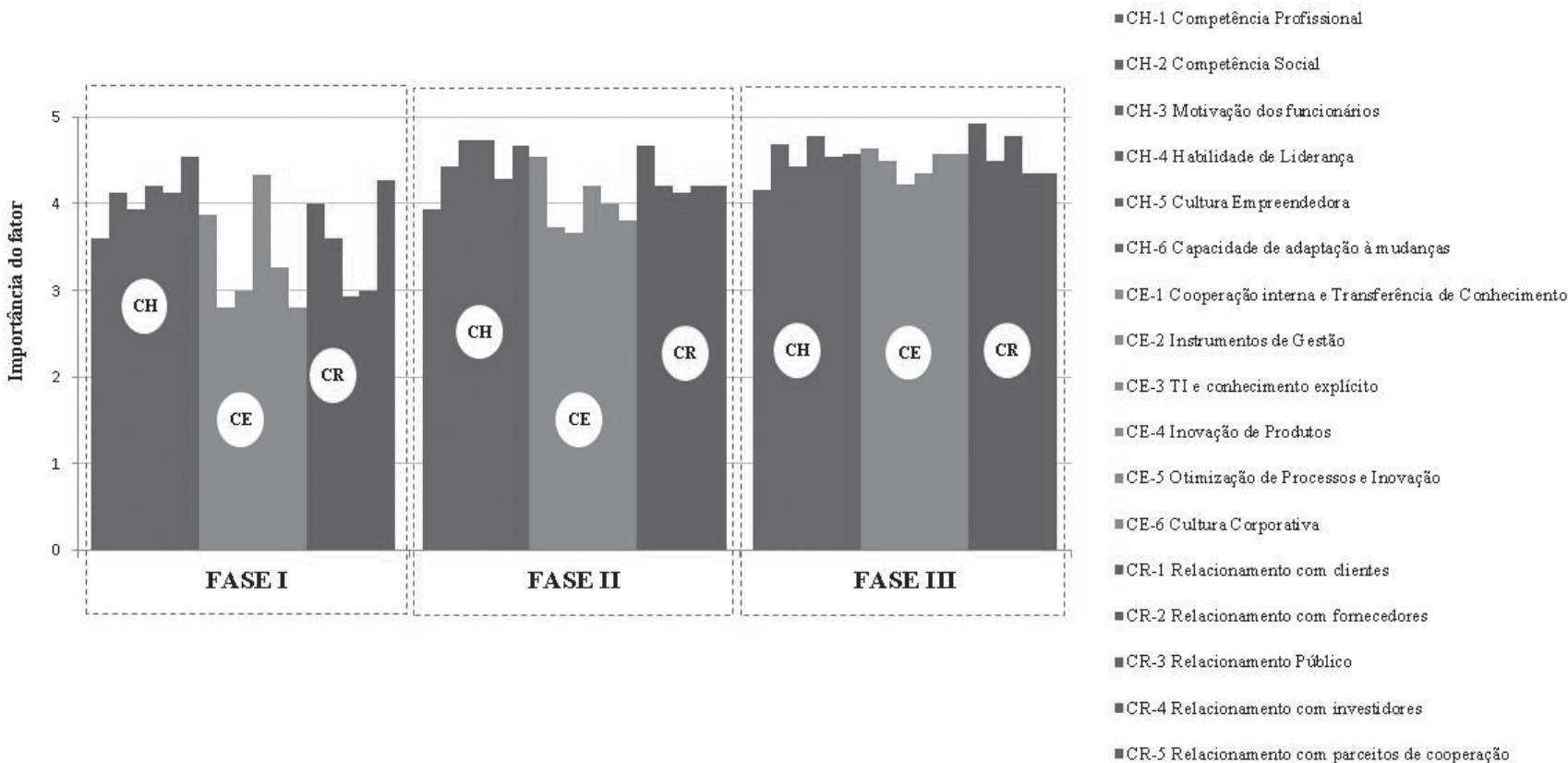

Figura 4.1. Resultados da avaliação da importância dos fatores de sucesso nas fases de evolução da startup. (Fonte: autores)

$\mathrm{Na}$ fase II, também destacam-se os fatores do capital humano, porém nota-se um aumento na importância dos fatores do capital estrutural e relacional na fase que representa o posicionamento do(s) produto(s) da empresa no mercado. Já a fase III apresenta médias de notas mais altas em todos os fatores e maior uniformidade quanto à importância das três dimensões do capital intelectual avaliadas sob a ótica dos empresários, sugerindo que esses fatores deveriam estar mais desenvolvidos na empresa, na fase em que ela inicia sua consolidação no mercado.

Em uma perspectiva diferente de apresentação dos dados, reforça-se a importância do capital humano nos cinco primeiros anos que abrangem as três fases, sem grandes variações, nas fases II e III, segundo a avaliação dos empresários. Para o capital estrutural e capital relacional nota-se que suas relevâncias são mais significativas nas fases II e III.
De acordo com os dados obtidos da avaliação dos empresários, observa-se que pelos valores de variância e desvios padrão, os resultados das fases II e III apresentam menor dispersão da população dos dados e, portanto, menor desvio em relação à média. Este resultado indica maior uniformidade nas avaliações dos empresários quanto às notas dadas para representar a importância dos fatores do capital intelectual nestas fases de vida das empresas.

\section{Conclusões}

Com a aplicação do método proposto, o objetivo foi obter um cenário para identificação de quais os tipos de capital intelectual que predominam em cada fase de evolução de uma empresa startup de base tecnológica, segundo a visão de empresários desse tipo de empresa. A identificação dos fatores de sucesso, mais significativos para a avaliação desse cenário, também foi considerada 


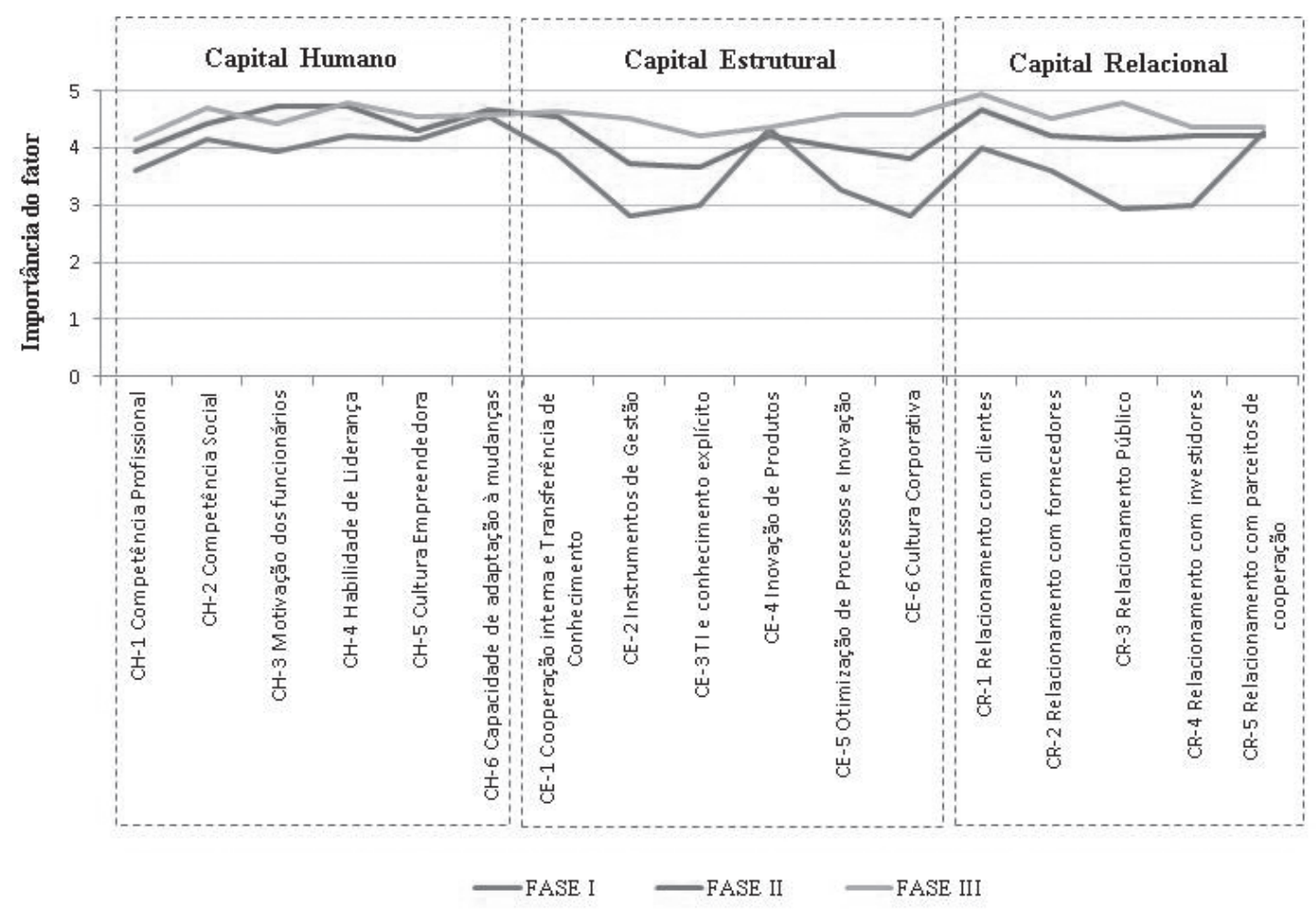

Figura 4.2. Avaliação da importância dos fatores de sucesso do capital intelectual

dentro dos resultados esperados. Esses dados são de fundamental relevância para a pesquisa, uma vez que trazem informações de como a combinação de ativos tangíveis e intangíveis e formas de desenvolvê-los podem influenciar no sucesso dessas empresas, principalmente, em seus primeiros, e mais críticos, anos de existência.

Os resultados apresentados mostram que há uma tendência de posicionar o capital humano nas duas primeiras fases da empresa como grande diferencial, desde o início do negócio, e a grande importância do capital relacional na fase III, de consolidação da empresa no mercado. O método proposto pode ser aplicado a outros cenários de empresas, bem como gerar quadros comparativos do capital intelectual por setores industriais em um mesmo tamanho de empresa.

A partir do trabalho apresentado, novos estudos para desenvolvimento do capital intelectual, baseado na identificação das necessidades observadas pelo tamanho ou segmento do negócio, podem ser desenvolvidos, possibilitando a inserção de novos conhecimentos acerca dos ativos intangíveis como diferencial no sucesso de empresas.

\section{Referências}

1. Baum, J. A. C., Calabrese, T., Silverman, B. S. Don't go it alone: Alliance netwrok composition and startups' performance in canadian biotechnology. Strategic Management Journal 21: 267294, 2000.

2. Blank, S. Why the Lean Startup changes everything. Harvard Business Review, v. 91. n. 5, 2013.

3. Dornelas, J. C. A. Empreendedorismo: transformando ideias em negócios. 3.ed. Rio de Janeiro: Elsevier, 2008.

4. Edital SENAI SESI de Inovação 2014. Serviço Nacional de Aprendizagem Industrial, Serviço Social da Indústria. Brasília: SENAI, 2014. Disponível em http://www.portaldaindustria.com. br/senai/iniciativas-senai/programas/edital-de-inovacao/2014/. 
5. FINEP Superintendência de Estudos e Estratégias Setoriais . Categorização das Empresas Clientes Finep. Rio de janeiro, 2000. Disponível em

6. http://www.finep.gov.br/o_que_e_a_finep/conceitos_ ct.asp\#indices

7. Freeman J., Engel, J. S. Models of Innovation: Startups and Mature Corporations. California Management Review. Vol.50 $\mathrm{n}^{\circ}$ 1. 2007

8. Hormiga, E., Batista-Canino, R. M., Sánchez-Medina, A.. The hole of intellectual capital in the success of new ventures. Springer. 2010.

9. Machado, E. Modelo de Análise da Influência do Capital Intelectual no Sucesso de Startups Incubadas. Tese de doutorado. Universidade Federal de Santa Catarina. 2014.

10. Mertins, K., Alwert, K., Will, M. Measuring Intellectual Capital in European SME. Proceedings of I-KNOW, Austria, 2006.

11. Mertins, K., Wang, W., Will, M. InCaS: Intellectual Capital Management in European SME - Its Strategic Relevance and the Importance of its Certification. The Electronic Journal of Knowledge Management. Vol $7 \mathrm{~N}^{\circ} 1$. 2009. Disponível em www. ejkm.com

12. Mertins, K.; Will, M.. A Consistent Assessment of Intellectual Capital in SMEs InCaS: Intellectual Capital Statement - Made in Europe. The Electronic Journal of Knowledge Management. Vol 5, $4^{\text {a }}$ Ed. 2007. Disponível em: www.ejkm.com

13. Peña, I. Intellectual capital and business start-up success. Journal of Intellectual Capital, v. 3, n. 2, 2002.

14. Revista Exame. O que é uma startup? Por GITARY, Y. 2010. http://exame.abril.com.br/pme/noticias/o-que-e-uma-startup. Acesso em 03/04/2015

15. Ribeiro, G. O. LEAN STARTUP: Análise exploratória sobre sua utilização por novas empresas brasileiras. Dissertação, FGV EASP. 2014.

16. Ries, Eric. A Startup Enxuta: como os empreendedores atuais utilizam a inovação contínua para criar empresas extremamente bem-sucedidas. São Paulo: Leya Brasil, 2012

17. Stewart, T. A. Capital Intelectual, a nova vantagem competitiva das empresas. $11^{\mathrm{a}}$ Ed. Rio de Janeiro: Campus, 1998.

18. Williamson, O. E. Markets and Hierarchies: Analysis and Antitrust Implication. Free Press. 1975

\section{Vanessa V. P. Canhete ${ }^{1^{*}}$, Jefferson O. Gomes ${ }^{2}$, Marcelo F. Prim', Juliana G. Ulliana' \& Germana A. B. Zapata $^{1}$}

${ }^{1}$ SENAI Departamento Nacional, Unidade de Inovação e Tecnologia, Brasília - DF

${ }^{2}$ Instituto Tecnológico de Aeronáutica. Praça Marechal Eduardo Gomes, 50 - Vila das Acácias. São Jose dos Campos, CEP: 12228-615, SP.

*E-mail: vcanhete@ita.br 